\title{
SOME PROPERTIES OF GENERALIZED 2-D MIRROR IMAGE AND ANTI MIRROR IMAGE POLYNOMIALS
}

\author{
V. Ramachandran \\ Electrical and \\ Computer Engineering \\ Concordia University \\ Montreal, Canada, H3G 1M8 \\ kamala@ece.concordia.ca
}

\author{
Ravi P. Ramachandran \\ Electrical and \\ Computer Engineering \\ Rowan University \\ Glassboro, NJ, 08028 \\ ravi@rowan.edu
}

\section{S. Gargour}

\author{
Electrical Engineering \\ Ecole Technologie Superieure \\ Montreal, Canada, H2T 2C8 \\ gargour@ele.etsmtl.ca
}

\begin{abstract}
This paper considers a new generalized method of formulating 2-D mirror-image polynomials (TMIP) and anti-mirror-image polynomials (TAMIP), starting from the 2-D denominator polynomial of a digital transfer function. The sets of polynomials are obtained such that the magnitude response of the starting transfer function is unaltered. The different cases for the TMIP and the TAMIP are presented along with how the TMIP and TAMIP can be used to test the minimum phase property of the 2-D polynomial they were derived from. The concept and new properties of a 2-D Discrete Reactance Function are introduced.
\end{abstract}

\section{INTRODUCTION}

In a previous paper [1], (1) the denominator polynomial of a 1$\mathrm{D}$ discrete transfer function is modified such that the magnitude of the frequency response remains unaltered and (2) the modified polynomial is decomposed into an infinite number of sums of mirror-image and anti-mirror image polynomials. The properties of these decompositions have been discussed in [1]. Some properties of multivariable mirror-image and anti-mirror image polynomials are discussed in [2][3]. In this paper, the new concept of generalized 2-D mirror-image and anti-mirror-image polynomials will be discussed. Specifically, a 2-D polynomial contained in the denominator of a transfer function is modified so that the magnitude of the frequency response remains unchanged (extension of the 1-D case). The modified 2-D denominator polynomial is decomposed into a sum of a two-variable mirror-image polynomial (TMIP) and a two-variable anti-mirror-image polynomial (TAMIP). The TMIP and TAMIP can be used to test for minimum phase of the original 2-D polynomial using the concept of inners. The definition and properties of a 2-D Discrete Reactance Function (2DDRF) are introduced. In this paper, the notation $\mathbf{Z}=\left(z_{1}, z_{2}\right)$ and $\mathbf{Z}^{-1}=\left(z_{1}^{-1}, z_{2}^{-1}\right)$ is used throughout.

\section{DEFINITION OF TMIP AND TAMIP}

Let $D(\mathbf{Z})$ be a two-variable polynomial given by

$$
D(\mathbf{Z})=\sum_{n_{1}=0}^{p_{1}} \sum_{n_{2}=0}^{p_{2}} d\left(n_{1}, n_{2}\right) z_{1}^{n_{1}} z_{2}^{n_{2}}
$$

$$
\begin{aligned}
& =\sum_{k=0}^{p_{1}} \alpha_{k}\left(z_{2}\right) z_{1}^{k} \\
& =\sum_{m=0}^{p_{2}} \beta_{m}\left(z_{1}\right) z_{2}^{m}
\end{aligned}
$$

This forms the denominator polynomial of a 2-D transfer function given by

$$
T(\mathbf{Z})=\frac{N(\mathbf{Z})}{D(\mathbf{Z})}
$$

The polynomial $D(\mathbf{Z})$ is modified to $D_{m}(\mathbf{Z})$ by multiplication by the factor $z_{1}^{q_{1}} z_{2}^{q_{2}}$ where $q_{1} \geq 0$ and $q_{2} \geq 0$. A modified transfer function $T_{m}(\mathbf{Z})$ with the same frequency response as $T(\mathbf{Z})$ results where

$$
T_{m}(\mathbf{Z})=\frac{N(\mathbf{Z})}{D_{m}(\mathbf{Z})}=\frac{N(\mathbf{Z})}{z_{1}^{q_{1}} z_{2}^{q_{2}} D(\mathbf{Z})}
$$

The polynomial $D_{m}(\mathbf{Z})$ is expressed as

$$
\begin{aligned}
D_{m}(\mathbf{Z}) & =\sum_{n_{1}=q_{1}}^{p_{1}+q_{1}} \sum_{n_{2}=q_{2}}^{p_{2}+q_{2}} d\left(n_{1}, n_{2}\right) z_{1}^{n_{1}} z_{2}^{n_{2}} \\
& =\sum_{k=q_{1}}^{p_{1}+q_{1}} z_{2}^{q_{2}} \alpha_{k}\left(z_{2}\right) z_{1}^{k} \\
& =\sum_{m=q_{2}}^{p_{2}+q_{2}} z_{1}^{q_{1}} \beta_{m}\left(z_{1}\right) z_{2}^{m}
\end{aligned}
$$

The TMIP is denoted as $M(\mathbf{Z})$ and is given as

$$
\begin{aligned}
2 M(\mathbf{Z}) & =D_{m}(\mathbf{Z})+z_{1}^{p_{1}+q_{1}} z_{2}^{p_{2}+q_{2}} D_{m}\left(\mathbf{Z}^{-1}\right) \\
& =D_{m}(\mathbf{Z})+z_{1}^{p_{1}} z_{2}^{p_{2}} D\left(\mathbf{Z}^{-1}\right)
\end{aligned}
$$

The TAMIP is denoted as $A(\mathbf{Z})$ and is given as

$$
\begin{aligned}
2 A(\mathbf{Z}) & =D_{m}(\mathbf{Z})-z_{1}^{p_{1}+q_{1}} z_{2}^{p_{2}+q_{2}} D_{m}\left(\mathbf{Z}^{-1}\right) \\
& =D_{m}(\mathbf{Z})-z_{1}^{p_{1}} z_{2}^{p_{2}} D\left(\mathbf{Z}^{-1}\right)
\end{aligned}
$$

The above development is an extension of the 1-D case discussed in [1]. Both the TMIP and the TAMIP can be formed for any 2-D polynomial. However, it it most useful to develop the TMIP and TAMIP for a minimum phase polynomial since the transfer functions $T(\mathbf{Z})$ and $T_{m}(\mathbf{Z})$ will be BIBO stable. Also, the new theorems presented in the sequel assume a minimum phase polynomial. 


\subsection{Distinct Cases for TMIP}

The various cases for the TMIP arranged in the variable $z_{1}$ are given below. Similar cases exist when the TMIP is arranged in $z_{2}$. These expressions can be obtained by algebraic manipulation. The coefficient conditions, given for each case, are in the form of an allpass transfer function.

Case 1: When $q_{1}=p_{1}+1$, the TMIP is

$$
\begin{aligned}
2 M(\mathbf{Z}) & =z_{1}^{p_{1}+1} z_{2}^{q_{2}} \sum_{k_{1}=0}^{p_{1}} \alpha_{k_{1}}\left(z_{2}\right) z_{1}^{k_{1}} \\
& +z_{2}^{p_{2}} \sum_{k_{2}=0}^{p_{1}} \alpha_{k_{2}}\left(z_{2}^{-1}\right) z_{1}^{p_{1}-k_{2}}
\end{aligned}
$$

The coefficient condition is

$$
\frac{\text { coefficient of } z_{1}^{i}}{\text { coefficient of } z_{1}^{2 p_{1}+1-i}}=\frac{z_{2}^{p_{2}} \alpha_{p_{1}-i}\left(z_{2}^{-1}\right)}{z_{2}^{q_{2}} \alpha_{p_{1}-i}\left(z_{2}\right)}
$$

for $i=1,2, \cdots, p_{1}$.

Case 2: When $q_{1}=p_{1}+1+x_{1}$ and $x_{1}>0$, the TMIP is

$$
\begin{aligned}
2 M(\mathbf{Z}) & =z_{1}^{p_{1}+1+x_{1}} z_{2}^{q_{2}} \sum_{k_{1}=0}^{p_{1}} \alpha_{k_{1}}\left(z_{2}\right) z_{1}^{k_{1}} \\
& +z_{2}^{p_{2}} \sum_{k_{2}=0}^{p_{1}} \alpha_{k_{2}}\left(z_{2}^{-1}\right) z_{1}^{p_{1}-k_{2}}
\end{aligned}
$$

The coefficient condition is

$$
\frac{\text { coefficient of } z_{1}^{i}}{\text { coefficient of } z_{1}^{2 p_{1}+1-i}}=\frac{z_{2}^{p_{2}} \alpha_{p_{1}-i}\left(z_{2}^{-1}\right)}{z_{2}^{q_{2}} \alpha_{p_{1}-i}\left(z_{2}\right)}
$$

for $i=1,2, \cdots, p_{1}$. For the TMIP, there are $x_{1}$ terms equal to zero. These terms should not be taken into account for determining the coefficient condition.

Case 3: When $q_{1}=p_{1}+1-x_{1}$ and $x_{1}>0$, the TMIP is

$$
\begin{aligned}
2 M(\mathbf{Z}) & =z_{1}^{p_{1}+1-x_{1}} z_{2}^{q_{2}} \sum_{k_{1}=0}^{p_{1}} \alpha_{k_{1}}\left(z_{2}\right) z_{1}^{k_{1}} \\
& +z_{2}^{p_{2}} \sum_{k_{2}=0}^{p_{1}} \alpha_{k_{2}}\left(z_{2}^{-1}\right) z_{1}^{p_{1}-k_{2}}
\end{aligned}
$$

The coefficient condition is

$$
\frac{\text { coefficient of } z_{1}^{i}}{\text { coefficient of } z_{1}^{2 p_{1}+1-i}}=\frac{z_{2}^{p_{2}} \alpha_{p_{1}-i}\left(z_{2}^{-1}\right)}{z_{2}^{q_{2}} \alpha_{p_{1}-i}\left(z_{2}\right)}
$$

for $i=1,2, \cdots, q_{1}$ and

$$
\frac{\text { coefficient of } z_{1}^{i}}{\text { coefficient of } z_{1}^{2 p_{1}+1-i}}=\frac{z_{2}^{p_{2}} \alpha_{i}\left(z_{2}^{-1}\right)+z_{2}^{q_{2}} \alpha_{p_{1}-q_{1}-i}\left(z_{2}\right)}{z_{2}^{q_{2}} \alpha_{p_{1}}\left(z_{2}\right)+z_{2}^{p_{2}} \alpha_{p_{1}-q_{1}-i}\left(z_{2}^{-1}\right)}
$$

for $i=q_{1}+1, \cdots, p_{1}+1-q_{1}$.

\subsection{Distinct Cases for TAMIP}

The various cases for the TAMIP arranged in the variable $z_{1}$ are given below. Similar cases exist when the TAMIP is arranged in $z_{2}$. As for the TMIP, the coefficient conditions are in the form of an allpass transfer function.

Case 1: When $q_{1}=p_{1}+1$, the TAMIP is

$$
\begin{aligned}
2 A(\mathbf{Z}) & =z_{1}^{p_{1}+1} z_{2}^{q_{2}} \sum_{k_{1}=0}^{p_{1}} \alpha_{k_{1}}\left(z_{2}\right) z_{1}^{k_{1}} \\
& -z_{2}^{p_{2}} \sum_{k_{2}=0}^{p_{1}} \alpha_{k_{2}}\left(z_{2}^{-1}\right) z_{1}^{p_{1}-k_{2}}
\end{aligned}
$$

The coefficient condition is

$$
\frac{\text { coefficient of } z_{1}^{i}}{\text { coefficient of } z_{1}^{2 p_{1}+1-i}}=(-1) \frac{z_{2}^{p_{2}} \alpha_{p_{1}-i}\left(z_{2}^{-1}\right)}{z_{2}^{q 2} \alpha_{p_{1}-i}\left(z_{2}\right)}
$$

for $i=1,2, \cdots, p_{1}$.

Case 2: When $q_{1}=p_{1}+1+x_{1}$ and $x_{1}>0$, the TAMIP is

$$
\begin{aligned}
2 A(\mathbf{Z}) & =z_{1}^{p_{1}+1+x_{1}} z_{2}^{q_{2}} \sum_{k_{1}=0}^{p_{1}} \alpha_{k_{1}}\left(z_{2}\right) z_{1}^{k_{1}} \\
& -z_{2}^{p_{2}} \sum_{k_{2}=0}^{p_{1}} \alpha_{k_{2}}\left(z_{2}^{-1}\right) z_{1}^{p_{1}-k_{2}}
\end{aligned}
$$

The coefficient condition is

$$
\frac{\text { coefficient of } z_{1}^{i}}{\text { coefficient of } z_{1}^{2 p_{1}+1-i}}=\frac{z_{2}^{p_{2}} \alpha_{p_{1}-i}\left(z_{2}^{-1}\right)}{z_{2}^{q_{2}} \alpha_{p_{1}-i}\left(z_{2}\right)}
$$

for $i=1,2, \cdots, p_{1}$. For the TAMIP, there are $x_{1}$ terms equal to zero. These terms should not be taken into account for determining the coefficient condition.

Case 3: When $q_{1}=p_{1}+1-x_{1}$ and $x_{1}>0$, the TMIP is

$$
\begin{aligned}
2 A(\mathbf{Z}) & =z_{1}^{p_{1}+1-x_{1}} z_{2}^{q_{2}} \sum_{k_{1}=0}^{p_{1}} \alpha_{k_{1}}\left(z_{2}\right) z_{1}^{k_{1}} \\
& -z_{2}^{p_{2}} \sum_{k_{2}=0}^{p_{1}} \alpha_{k_{2}}\left(z_{2}^{-1}\right) z_{1}^{p_{1}-k_{2}}
\end{aligned}
$$

The coefficient condition is

$$
\frac{\text { coefficient of } z_{1}^{i}}{\text { coefficient of } z_{1}^{2 p_{1}+1-i}}=(-1) \frac{z_{2}^{p_{2}} \alpha_{p_{1}-i}\left(z_{2}^{-1}\right)}{z_{2}^{q_{2}} \alpha_{p_{1}-i}\left(z_{2}\right)}
$$

for $i=1,2, \cdots, q_{1}$ and

$\frac{\text { coefficient of } z_{1}^{i}}{\text { coefficient of } z_{1}^{2 p_{1}+1-i}}=\frac{-z_{2}^{p_{2}} \alpha_{i}\left(z_{2}^{-1}\right)+z_{2}^{q_{2}} \alpha_{p_{1}-q_{1}-i}\left(z_{2}\right)}{z_{2}^{q_{2}} \alpha_{p_{1}}\left(z_{2}\right)+-z_{2}^{p_{2}} \alpha_{p_{1}-q_{1}-i}\left(z_{2}^{-1}\right)}$

for $i=q_{1}+1, \cdots, p_{1}+1-q_{1}$. 


\section{2-D DISCRETE REACTANCE FUNCTION}

The 2-D Discrete Reactance Function (2DDRF) is defined as the ratio of the TMIP to the TAMIP as given by

$$
R(\mathbf{Z})=\frac{M(\mathbf{Z})}{A(\mathbf{Z})}
$$

where $M(\mathbf{Z})$ is defined in Eq. (10) and $A(\mathbf{Z})$ is defined in Eq. (12). Note that $1 / R(\mathbf{Z})$ is also a $2 \mathrm{DDRF}$. The eoncept of a $2 \mathrm{DDRF}$ is an extension of the one-dimensional DRF discussed in [1]. The following new theorems are introduced and assume that $D(\mathbf{Z})$ is minimum phase.

Theorem 1: For $\left|z_{1}\right|=1$ and $z_{2}=r_{2} e^{j \omega_{2}}$, we have

$$
\operatorname{Re}|R(\mathbf{Z})| \text { is }\left\{\begin{array}{ll}
<0 & \text { for } r_{2}<1 \\
=0 & \text { for } r_{2}=1 \\
>0 & \text { for } r_{2}>1
\end{array},\right.
$$

where $\operatorname{Re}()$ denotes the real part.

Proof: The proof is similar to Theorem 2 in [1]. Let

$$
H(\mathbf{Z})=\frac{z_{1}^{p_{1}} z_{2}^{p_{2}} D\left(\mathbf{Z}^{-1}\right)}{D_{m}(\mathbf{Z})}
$$

Then, it follows that

$$
R(\mathbf{Z})=\frac{1+H(\mathbf{Z})}{1-H(\mathbf{Z})}
$$

When $\left|z_{1}\right|=1, H(\mathbf{Z})$ represents a stable allpass function in $z_{2}$ having complex coefficients. It is known that an allpass function in a single variable $z_{2}=r_{2} e^{j \omega_{2}}$ has a magnitude greater than 1 within the unit circle $\left(r_{2}<1\right)$, equal to 1 on the unit circle $\left(r_{2}=1\right)$, and less than 1 outside the unit circle $\left(r_{2}>1\right)$. Suppose $H(\mathbf{Z})=a+j b$ when $\left|z_{1}\right|=1$ and $z_{2}=r_{2} e^{j \omega_{2}}$. Then,

$$
\operatorname{Re}|R(\mathbf{Z})|=\frac{1-\left(a^{2}+b^{2}\right)}{(1-a)^{2}+b^{2}}
$$

When $r_{2}<1, a^{2}+b^{2}>1$ thereby establishing that $\operatorname{Re}|R(\mathbf{Z})|<$ 0 . Similarly, the conditions of the theorem for $r_{2}=1$ and $r_{2}>1$ are established.

Theorem 2: This is similar to Theorem 1 and states that for $\left|z_{2}\right|=1$ and $z_{1}=r_{1} e^{j \omega_{1}}$, we have

$$
\operatorname{Re}|R(\mathbf{Z})| \text { is } \begin{cases}<0 & \text { for } r_{1}<1 \\ =0 & \text { for } r_{1}=1 \\ >0 & \text { for } r_{1}>1\end{cases}
$$

When $\left|z_{1}\right|=1$ and $\left|z_{2}\right|=1$, (1) $R(\mathbf{Z})$ is complex and (2) from the results of Theorems 1 and 2, $R(\mathbf{Z})$ is a Positive Exterior Function (PRF) [4].

Theorem 3: When $\left|z_{1}\right|=1, R(\mathbf{Z})$ contains complex coefficients and its poles and zeros are simple, lie on the unit circle of the $z_{2}$ plane and interlace. A similar result holds when $\left|z_{2}\right|=1$. The proof follows from the preceding discussion. When the poles and zeros coincide, they form nonessential singularities of the second kind [5].

A similar result has been shown for a 2-D analog reactance function [6]. If one of the variables, say $s_{1}$, is fixed on the imaginary axis, the resulting analog reactance function has complex coefficients and its poles and zeros are simple, lie on the imaginary axis of the $s_{2}$ plane and interlace.

\section{NUMERICAL EXAMPLE}

An example [3] of a minimum phase polynomial is given by $D(\mathbf{Z})=$ $12 z_{1}^{2} z_{2}+6 z_{1}^{2}+10 z_{1} z_{2}+5 z_{1}+2 z_{2}+1$. In this case, $p_{1}=2$ and $p_{2}=1$. Taking $q_{1}=4$ and $q_{2}=1$, we get the TMIP as

$$
M(\mathbf{Z})=\sum_{n=0}^{6} M_{2 n}\left(z_{2}\right) z_{1}^{n}
$$

where

$$
\begin{aligned}
& M_{20}\left(z_{2}\right)=3 z_{2}+6 \\
& M_{21}\left(z_{2}\right)=2.5 z_{2}+5 \\
& M_{22}\left(z_{2}\right)=0.5 z_{2}+1 \\
& M_{23}\left(z_{2}\right)=0 \\
& M_{24}\left(z_{2}\right)=z_{2}^{2}+0.5 z_{2}=z_{2}^{2} M_{22}\left(z_{2}^{-1}\right) \\
& M_{25}\left(z_{2}\right)=5 z_{2}^{2}+2.5 z_{2}=z_{2}^{2} M_{21}\left(z_{2}^{-1}\right) \\
& M_{26}\left(z_{2}\right)=6 z_{2}^{2}+3 z_{2}=z_{2}^{2} M_{20}\left(z_{2}^{-1}\right)
\end{aligned}
$$

The TAMIP is given by

$$
A(\mathbf{Z})=\sum_{n=0}^{6} A_{2 n}\left(z_{2}\right) z_{1}^{n}
$$

where

$$
\begin{aligned}
& A_{20}\left(z_{2}\right)=-3 z_{2}-6 \\
& A_{21}\left(z_{2}\right)=-2.5 z_{2}-5 \\
& A_{22}\left(z_{2}\right)=-0.5 z_{2}-1 \\
& A_{23}\left(z_{2}\right)=0 \\
& A_{24}\left(z_{2}\right)=z_{2}^{2}+0.5 z_{2}=-z_{2}^{2} A_{22}\left(z_{2}^{-1}\right) \\
& A_{25}\left(z_{2}\right)=5 z_{2}^{2}+2.5 z_{2}=-z_{2}^{2} A_{21}\left(z_{2}^{-1}\right) \\
& A_{26}\left(z_{2}\right)=6 z_{2}^{2}+3 z_{2}=-z_{2}^{2} A_{20}\left(z_{2}^{-1}\right)
\end{aligned}
$$

The TMIP and TAMIP can also be written as a linear combination of powers of $z_{2}$. The TMIP is expressed as

$$
M(\mathbf{Z})=\sum_{n=0}^{2} M_{1 n}\left(z_{1}\right) z_{2}^{n}
$$

where

$$
\begin{aligned}
& M_{10}\left(z_{1}\right)=z_{1}^{2}+5 z_{1}+6 \\
& M_{11}\left(z_{1}\right)=3 z_{1}^{6}+2.5 z_{1}^{5}+0.5 z_{1}^{4}+0.5 z_{1}^{2}+2.5 z_{1}+3 \\
& M_{12}\left(z_{1}\right)=6 z_{1}^{6}+5 z_{1}^{5}+z_{1}^{4}=z_{1}^{6} M_{10}\left(z_{1}^{-1}\right)
\end{aligned}
$$

The TAMIP is expressed as

$$
A(\mathbf{Z})=\sum_{n=0}^{2} A_{1 n}\left(z_{1}\right) z_{2}^{n}
$$

where

$$
\begin{aligned}
& A_{10}\left(z_{1}\right)=-z_{1}^{2}-5 z_{1}-6 \\
& A_{11}\left(z_{1}\right)=3 z_{1}^{6}+2.5 z_{1}^{5}+0.5 z_{1}^{4}-0.5 z_{1}^{2}-2.5 z_{1}-3 \\
& A_{12}\left(z_{1}\right)=6 z_{1}^{6}+5 z_{1}^{5}+z_{1}^{4}=-z_{1}^{6} A_{10}\left(z_{1}^{-1}\right)
\end{aligned}
$$

Figure 1 shows a plot of the interlacing poles and zeros of $R(\mathbf{Z})$ in the $z_{1}$ plane when $z_{2}=e^{j 3 \pi / 4}$. 


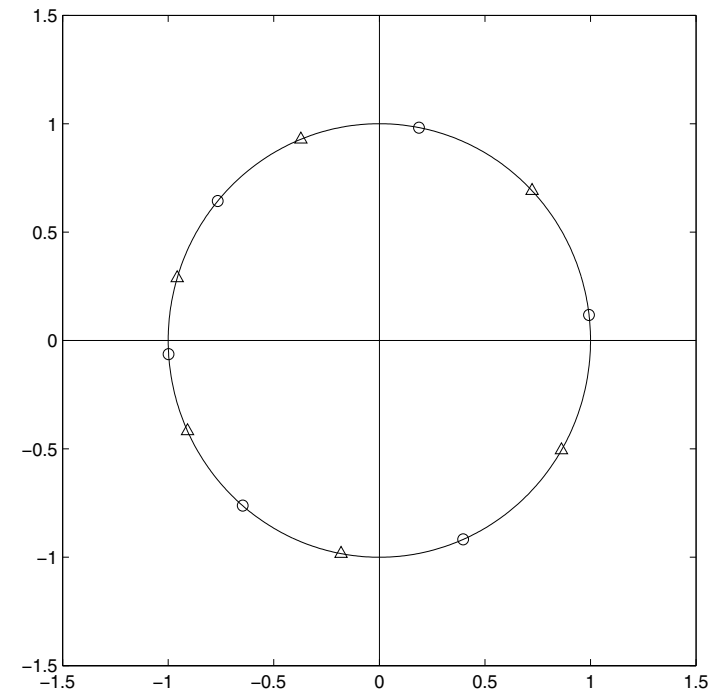

Figure 1: Poles and zeros of $R(\mathbf{Z})$ in the $z_{1}$ plane when $z_{2}=$ $e^{j 3 \pi / 4}$. The zeros are denoted by a $\bigcirc$. The poles are denoted by a $\Delta$.

\section{MINIMUM PHASE CONSIDERATIONS}

As mentioned earlier, (1) the theorems given above assume a minimum phase polynomial and (2) the TMIP and TAMIP can be formed for any polynomial. In fact, the TMIP and TAMIP can be used to test for minimum phase using the concept of inners [3][7][8]. The matrix of inners, T, can be formed using the TMIP and TAMIP written as a linear combination of powers of $z_{1}$ or $z_{2}$ [3]. For the example given above with the TMIP and TAMIP written as a combination of powers of $z_{2}, \mathbf{T}$ has dimension 4 as given by

$$
\mathbf{T}=\left[\begin{array}{cccc}
A_{12} & A_{11} & 0 & A_{10} \\
0 & A_{12} & A_{10} & A_{11} \\
0 & M_{12} & M_{10} & M_{11} \\
M_{12} & M_{11} & 0 & M_{10}
\end{array}\right]
$$

There are two inners, namely, $\mathbf{T}$ itself and the submatrix $\mathbf{T}_{1}$ given by

$$
\mathbf{T}_{1}=\left[\begin{array}{ll}
A_{12} & A_{10} \\
M_{12} & M_{10}
\end{array}\right]
$$

Two conditions for minimum phase are established since

$$
\frac{|\mathbf{T}|}{3 z_{1}^{12}}=\left[\left(6 z_{1}^{2}+5 z_{1}+1\right)\left(6 z_{1}^{-2}+5 z_{1}^{-1}+1\right)\right]^{2}
$$

and

$$
\frac{\left|\mathbf{T}_{1}\right|}{2 z_{1}^{6}}=\left(6 z_{1}^{2}+5 z_{1}+1\right)\left(6 z_{1}^{-2}+5 z_{1}^{-1}+1\right)
$$

(the symbol $|$.$| denotes determinant) are both positive when \left|z_{1}\right|=$ 1. The other condition is that the function of $z_{1}$ corresponding to the highest power of $z_{2}$ of $D(\mathbf{Z})$ should be a Schur polynomial [9] in $z_{1}$. In the example, the highest power of $z_{2}$ is 1 and the corresponding function, namely, $12 z_{1}^{2}+10 z_{1}+2$ is a Schur polynomial. If the TMIP and TAMIP are written as a linear combination of powers of $z_{1}$, the dimension of the matrix of inners is 8 thereby imposing more computation.

\section{SUMMARY AND CONCLUSIONS}

This paper considers the generalization of two-variable mirrorimage and anti-mirror-image polynomials. It is shown that a large number of possibilities exist and these are obtained by the denominator polynomial of a 2-D transfer function whose magnitude response remains unchanged even though the multiplying factor $z_{1}^{q_{1}} z_{2}^{q_{2}}$ is imposed. This is similar to the generalization carried out for the 1-D case [1]. The 2-D case gives rise to a large number of 2-D discrete reactance functions (2DDRF) that are obtained from a minimum phase 2-D polynomial. This is in contrast to the 1-D case where only two types of 1-D discrete reactance functions are defined such that the degree difference between the numerator and the denominator can be either zero or one. One important property of the 2DDRF is that for a point on the unit circle of one of the variables, the poles and zeros of the 2DDRF are simple and interlace on the unit circle in the plane of the other variable. It is also shown that the minimum phase property of a 2-D polynomial can be established using a matrix of inners based on the TMIP and the TAMIP.

\section{REFERENCES}

1. V. Ramachandran, R. P. Ramachandran and C. S. Gargour, "Some Properties of the Z-domain Continued Fraction Expansion of 1-D Discrete Reactance Functions", IEEE Int. Symp. on Circuits and Systems, Sydney, Australia, May 2001, pp. II-841-II-844.

2. V. Ramachandran and M. Ahmadi, "Multivariable MirrorImage and Anti-Mirror-Image Polynomials obtained by $\mathrm{Bi}$ linear Transformations", IEEE Transactions on Circuits and Systems, Vol.CAS-34, No. 9, pp. 1088-1090, September 1987.

3. V. Ramachandran and M. Ahmadi, "Some Properties of Multivariable Mirror-Image and Anti-Mirror-Image Polynomials obtained by the Bilinear Transformation of Hurwitz Polynomials", IEEE Transactions on Circuits and Systems, Vol. 37, No. 6, pp. 828-831, June 1990.

4. A. M. Davis, "Realizability preserving transformations for digital and analog filters", Journal of Franklin Institute, Vol. 311, pp. 111-121, February 1981.

5. V. Ramachandran and C. S. Gargour, "Generation of Very Strict Hurwitz Polynomials and Applications to 2-D Filter Design", in Multidimensional Systems: Signal Processing and Modeling Techniques, Academic Press Inc., Vol. 69, pp.211-254, 1995.

6. T. Koga, "Synthesis of Passive n-ports with prescribed TwoVariable Reactance Matrices", IEEE Transactions on Circuit Theory, Vol. CT-13, No. 1, pp.31-52, March 1966.

7. E. I. Jury, Inners and Stability of Dynamic Systems, Krieger, 1982.

8. N. K. Bose, Applied Multidimensional System Theory, Van Nostrand Reinhold Co., 1982.

9. M. Marden, Geometry of Polynomials, American Mathematical Society, 1966. 